\title{
Preparation of Titanium Alloys through Molten Salt Electrolysis of Solid Oxides for Medical Implants
}

\author{
Jie $\mathrm{Li}^{1, \mathrm{a}, *}$, Tian $\mathrm{Wu}^{1,2,3, \mathrm{~b}, *}$ Shi He${ }^{1, \mathrm{c}}$, Gongxuan Chen ${ }^{2, \mathrm{~d}}$ and Xianbo Jin ${ }^{3, \mathrm{e}, *}$ \\ ${ }^{1}$ School of Physics and Mechanical \& Electronical Engineering, Hubei University of Education, \\ Wuhan 430205, China \\ ${ }^{2}$ Chemistry and Biology Science College, Hubei University of Education, Wuhan 430205, China \\ ${ }^{3}$ College of Chemistry and Molecular Sciences, Wuhan University, Wuhan 430072, China \\ a lijie@hue.edu.cn, ${ }^{\mathrm{b}}$ twu@whu.edu.cn, ${ }^{\mathrm{c}}$ heshi199402@gmail.com, ${ }^{\mathrm{d}}$ chengongxuan@ hue.edu.cn, \\ exbjin@whu.edu.cn \\ * Jie Li, Tian Wu and Xianbo Jin
}

Keywords: electrochemical reduction; medical implants; titanium alloys; molten salt

\begin{abstract}
A simple, cost-efficient, energy-saving, electrochemical process is developed to synthesize the biomedical Ti-based metal-containing Group VB (V, Nb, Ta) implant materials in the small size at $900{ }^{\circ} \mathrm{C}$. The anhydrous $\mathrm{CaCl}_{2}$ and solid oxides were used as the salt melt medium and raw reactants, respectively. The mechanism of the reduction process was discussed by constant cell voltage electrolysis of different time periods. The as-prepared samples, such as Ti-12.5Zr2.5Nb-2.5Ta, were characterized by SEM, EDX, elemental and biological analysis (including cell compatibility test, etc.). Because of the green synthesis, non-toxicity, and good biocompatibility, Ti12.5Zr-2.5Nb-2.5Ta exhibited promise for medical implant materials.
\end{abstract}

\section{Introduction}

The biomedical implant materials with high biocompatibility and mechanical properties have attracted extensive research interest in the past decades due to the aging of human society, the global climate instability and the large unpredictable disasters. Among the various biomedical implant materials, the metal materials in the clinical application have always been proven to be one of the most effective materials owing to their excellent properties, including mechanical properties, fatigue resistance, biocompatibility, corrosion resistance, low cost, etc [1]. The first material for implantation was the stainless steel 18-8 (standard grade No.302). On this basis, the scientists have developed a new stainless steel named 316 as a substitute material. Beginning from the 1950s, the carbon content in steel 316 have been reduced by the scientists to increase its resistance to corrosion in chloride solution, which is named as 316L stainless steel [2]. In terms of 316L stainless steel material, the relatively cheaper synthetic means reduced the costs for many manufacturers.

Extensive researches demonstrated that Ti-6Al-4V and the titanium have better performances in medical implant application compared with the stainless steel due to its good corrosion resistance, excellent elastic modulus and light density close to bone. However, it can not be ignored that the toxicity of vanadium has potential threat to human health. From the beginning of the last $90^{\text {th }}$ century, two kinds of novel $\alpha+\beta$ type alloys, Ti-5Al-2.5Fe and Ti-6Al-7Nb, began to be widely developed [3]. In this trend, Northwest Nonferrous Metal Research Institute and the Fourth Military Medical University in china also co-developed the Ti-2.5Al-2.5Mo-2.5Zr (TAMZ) alloys [1,4]. Because the TAMZ alloys could not cause cell toxicity and acute hemolytic reaction, it was expected to become a good dental biomaterials and surgical implants. However, the alloys containing element $\mathrm{Al}$ can lead to organ damage, causing osteomalacia, anemia, neurological disorders [5]. In recent years, the Northwest Nonferrous Metal Research Institute has developed a new medical titanium alloy: Ti-12.5Zr-2.5Nb-2.5Ta, referred as TZNT alloys. The alloy does not contain toxic elements (Al, V, Fe, etc.), which has better biomedical compatibility than Ti-6Al-4V, 
Ti-6Al-7Nb and other titanium-base alloys. Therefore, it was regarded as an excellent and new medical titanium alloy.

In addition to the stainless steel and some titanium alloys, Ni-Ti alloy is a good biomedical implant material, which is a shape memory alloy with low elastic modulus and good biocompatibility, applying the artificial heart, arterial stents, orthopedic implants and so on [2]. However, scientists have found the loosening and breakdown of metal implant material because the elastic modulus does not match with the body, resulting in secondary fractures from stress shielding in recent years. In addition, as a kind of biologically inert material, the biological activity of Ni-Ti alloy is relatively poor, as a result, it could be easily and loosely changed. Therefore, the scientists have taken some surface treatment approach for implant materials modification [1].

The industry has generally made use of the Melting-Rapid solidification method to synthesize the alloy materials in metallurgy [6], the preparation process involves some disadvantages, such as complex processes and high cost. Recently the method of molten electrolysis of solid oxide has been applied in the preparation of functional materials, including superconducting materials $\left(\mathrm{Nb}_{3} \mathrm{Sn}\right.$ $[7,8])$ and magnetic materials alloys $\left(\mathrm{Ni}_{2} \mathrm{MnGa}[19]\right)$. This method are highly attractive to many applications due to the costs, energy consumption and simplifying the process. we can directly set the ratio of the mixing oxide to control the alloy composition of electrolysis products without needing pure elemental metals as raw materials. So the molten electrolysis route of solid oxide has good prospects for development in industry.

Though large-scale industrialization in progress of FFC method has achieved grate achievements [10], seeking more suitable synthetic routs are the common goals for the FFC researchers. it is needed to adopt alternative ways to reduce energy consumption and support sustainable development in the metallurgical industry, which is in particular to simplify production steps as well as direct preparation of metal [11].

Herein we have prepared Ti-based biomedical materials, especially the popular TZNT series alloys, via the molten salt electrolysis method using mixed oxide solid as raw materials. The reaction mechanism was discussed by adjusting the molten salt electrolysis conditions. This operation showed high current efficiency and less energy consumption without the costly signal metal. In addition, the excellent results indicated the electro-reduction method to be indeed significant for replacing the Melting-Rapid solidification method. The as-obtained samples exhibited promise for medical implant materials due to their green synthesis, non-toxicity, and good biocompatibility.

\section{Experimental}

\subsection{Synthesis}

All the chemical reagents were of analytical grade and purchased from Aldrich. Firstly, the appropriate amounts of $\mathrm{TiO}_{2}, \mathrm{Nb}_{2} \mathrm{O}_{5}, \mathrm{Ta}_{2} \mathrm{O}_{5}, \mathrm{ZrO}_{2} 、 \mathrm{Al}_{2} \mathrm{O}_{3}, \mathrm{Fe}_{2} \mathrm{O}_{3}$ and $\mathrm{V}_{2} \mathrm{O}_{3}$ powders were mixed together at the molar ratio of Ti:Al:V = 90:6:4, Ti:Al:Nb=87:6:7, Ti:Ta=3:7, Ti:Al:Fe=92.5:5:2.5, V:Ti=4:1, Ti:Zr:Nb:Ta=82.5:12.5:2.5:2.5, respectively. Secondly, the mixture was finely milled with anhydrous alcohol for $7 \mathrm{~h}$. The samples prepared by milling was pressed at 7 $\mathrm{MPa}$ into cylindrical pellets, and then were sintered at $300{ }^{\circ} \mathrm{C}$ for $2 \mathrm{~h}$. Thirdly, The as-prepared pellets were wrapped with molybdenum wires to form an assembled cathode, while a graphite was used as the anode. Pre-electrolysis was performed at 2.2, 2.4, 2.6, 2.8 and 3.0 V to remove redoxactive impurities (such as $\mathrm{Mg}$ and $\mathrm{Zn}$ ) in the molten salt between a nickel foil cathode and a graphite rod anode, respectively. The electrolysis reactions were carried out at 900 and $950{ }^{\circ} \mathrm{C}$ for 10, 15, $20 \mathrm{~h}$ under constant voltage and a flow of argon (>99.999\%) in molten $\mathrm{CaCl}_{2}$, respectively.

\subsection{Measurement and characterization techniques}

The contrast commercial sample using as artificial joints was brought from the bone surgery center of Zhongnan Hospital of Wuhan University, for the sake of simplicity, we marked it as "Specimen M" in the following text. 
In order to avoid the interference to the result of cell compatibility due to the product surface roughness, the as-obtained samples were burnished by the sand paper, and then handled in the ethanol solution and distilled water by ultrasonic for 30 minutes, finally deposed in culture board after sterilization for the follow-up test.

By the use of primary cell culture techniques, human cancer tissues and cultured cancer cells were taken into test (A549 human lung carcinoma cell line), separately [12]. By means of the observation of phase contrast microscope, we could know that the variation of the morphology of the cell and the corresponding cell numbers represented for the trend of overall growth.

The electrolysis was controlled by a high-accuracy four-electrode battery testing system (Shenzhen Neware Electronic Ltd., PRC). Cyclic voltammograms (CVs) and potentiostatic electrolysis of solid mixed oxides in $\mathrm{CaCl}_{2}$ melt were recorded using the metallic (Mo) cavity electrode (MCE) and a computer-assisted IVIUM Electrochemical System (Ivium Technologies BV Company), respectively. A small amount of mixed oxide power was manually loaded into the double-cavity of the MCE, which was then inserted into the molten salt as working electrode. The graphite counter electrode and an $\mathrm{Ag} / \mathrm{Ag}^{+}$reference electrode $(10 \mathrm{~mol} \% \mathrm{AgCl}, 45 \mathrm{~mol} \% \mathrm{NaCl}$ and $45 \% \mathrm{~mol} \% \mathrm{KCl}$, sealed in a ceramic tube) were engaged in the electrochemical experiments.

The fine collected products were dried and characterized by an X-ray diffraction (SHIMADZU Lab XRD-6000 with $\mathrm{Cu} \mathrm{K} \alpha 1$, scanning rate of $6 \% \mathrm{~min}$ ), scanning electron microscopy (FEI Sirion Field Emission Gun SEM system) together with energy-dispersive X-ray analysis (EDX). Olympus DP72 was used for taking pictures of the Phase Contrast Upright Microscope. The oxygen content was determined by Inert Gas Fusion Oxygen Analysis (IGFOA, RO-416DR, LECO, USA).

\section{Results and discussion}

\subsection{Characteristic comparison of the products}

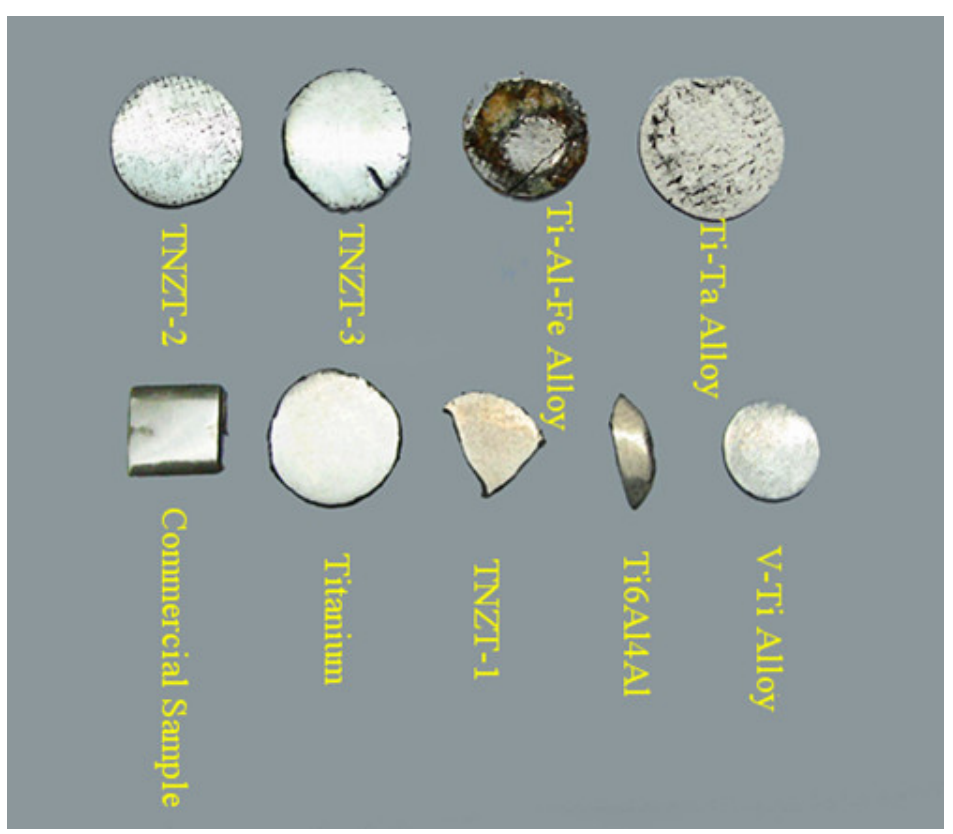

Fig 1 Selected the metal and alloy products after polished

The pure titanium, Ti-6Al-4V, Ta-30Ti, Ti-5Al-2.5Fe (referred to as TAF), V-20Ti, Ti-12.5Zr2.5Nb-2.5Ta (referred to as TZNT) alloys were prepared by constant cell voltage electrolysis at 2.2 $\mathrm{V}, 2.4 \mathrm{~V}, 2.6 \mathrm{~V}, 2.8 \mathrm{~V}$ and $3.0 \mathrm{~V}$ at 900 and $950{ }^{\circ} \mathrm{C}$, respectively. It is worth noting that we used TZNT-1, TZNT-2 and TZNT-3 to represent the different synthetic conditions of Ti-12.5Zr-2.5Nb2.5Ta. Specifically, TNTT- 1 was prepared at $900^{\circ} \mathrm{C}$ and $3.0 \mathrm{~V}$ for $20 \mathrm{~h}$, TNTT-2 was synthesized at $950{ }^{\circ} \mathrm{C}$ and $3.0 \mathrm{~V}$ for $20 \mathrm{~h}$, while TNTT-3 was synthesized at $950{ }^{\circ} \mathrm{C}$ and $3.0 \mathrm{~V}$ for $10 \mathrm{~h}$. Fig 1 displayed the appearance chart of the as-prepared samples as well as the commercial products after sanding treatment. As can be seen from the fig that the surface of Ti-5Al-2.5Fe alloy got rusty after 
washing, besides, the cracks were fined in the surface of the Ta-30Ti alloy. However, the pure titanium, TZNT and Ti-6Al-4V were still in the good appearance, which were very close to Specimen $M$. The as-obtained samples with good performance and Specimen $M$ with the appropriate sizes were used for cell culture. For the sake of simplicity, the list of all the samples is numbered in Table 1.

Table1 The list of all the samples

\begin{tabular}{|c|c|}
\hline Sample & Notification \\
\hline Specimen M & S1 \\
\hline TNZT-1 & S2 \\
\hline VTI & S3 \\
\hline TNZT-3 & S6 \\
\hline Pure Titanium & S7 \\
\hline Back side of TNZT-2 (Contrast) & S8 \\
\hline Back side of TNZT-3 (Contrast) & S9 \\
\hline Back side of TNZT-1 (Contrast) & S10 \\
\hline Back side of imported materials (Contrast) & S11 \\
\hline Specimen M & \\
\hline
\end{tabular}

\subsection{Cell toxicity test of metallic materials}

\subsubsection{Morphology of the cells around metallic materials}

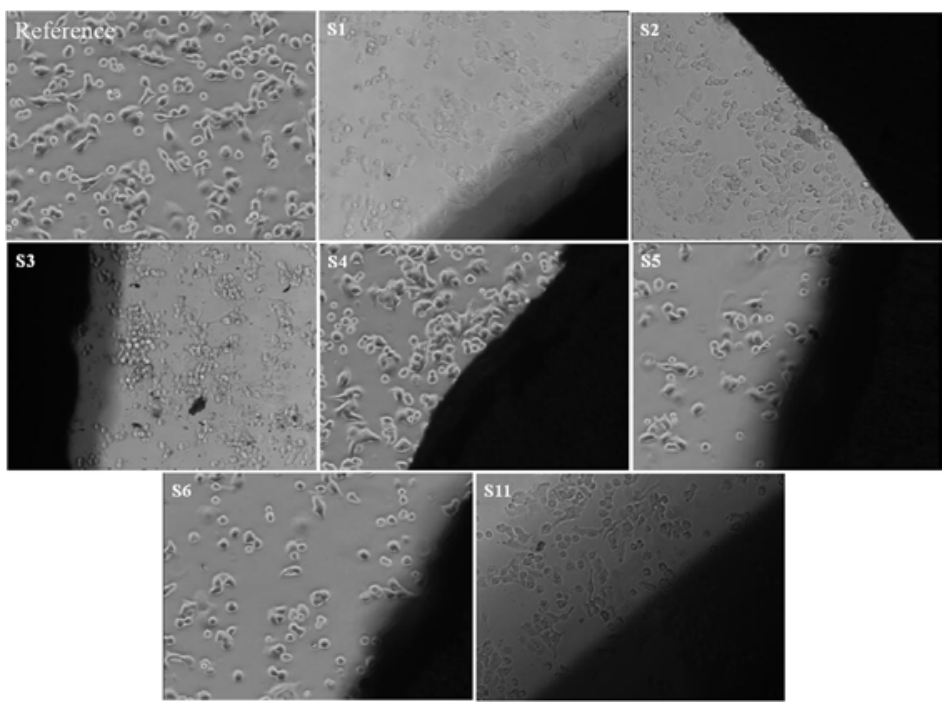

Fig 2 SEM images of the cells around the metal and alloy materials in the culture medium.

As can be seen from Fig 2, the cells around Specimen M exhibited better morphology as well as they were distributed more uniformly as compared with the cells in the body cell solution. In addition, the morphology of the cell around three TNZT materials prepared by different preparation conditions were similar to Specimen M. However, the cells surrounding the titanium metal were scattered obviously. More seriously, the cell clusters around the vanadium titanium alloy had nearly died due to dissolution of toxic vanadium ions from vanadium titanium alloy, causing physical harm for the cell [13]. Therefore, we can get the following conclusions for the cytotoxicity of materials:

Specimen $\mathrm{M}<\mathrm{TNZT}<$ metal titanium $<<$ vanadium titanium alloy

\subsubsection{Cell state diagram of the surface of metal materials}

Fig 3 showed that the morphologies of the cells grown on the surface of Specimen $M$ were very well. The morphologies of the cells grown on the surface of the three TNZT materials were similar to that of Specimen M. However, few of the cells was found on the pure titanium surface. More 
seriously, no cell could function on the surface of the vanadium titanium alloy due to dissolution of toxic vanadium ions resulting in physical harm for the cell. Based on the above observation, for the biocompatibility of the materials, the following conclusions could be as follows:

Specimen $\mathrm{M}>\mathrm{TNZT}>>$ metal titanium $>>$ vanadium titanium alloy

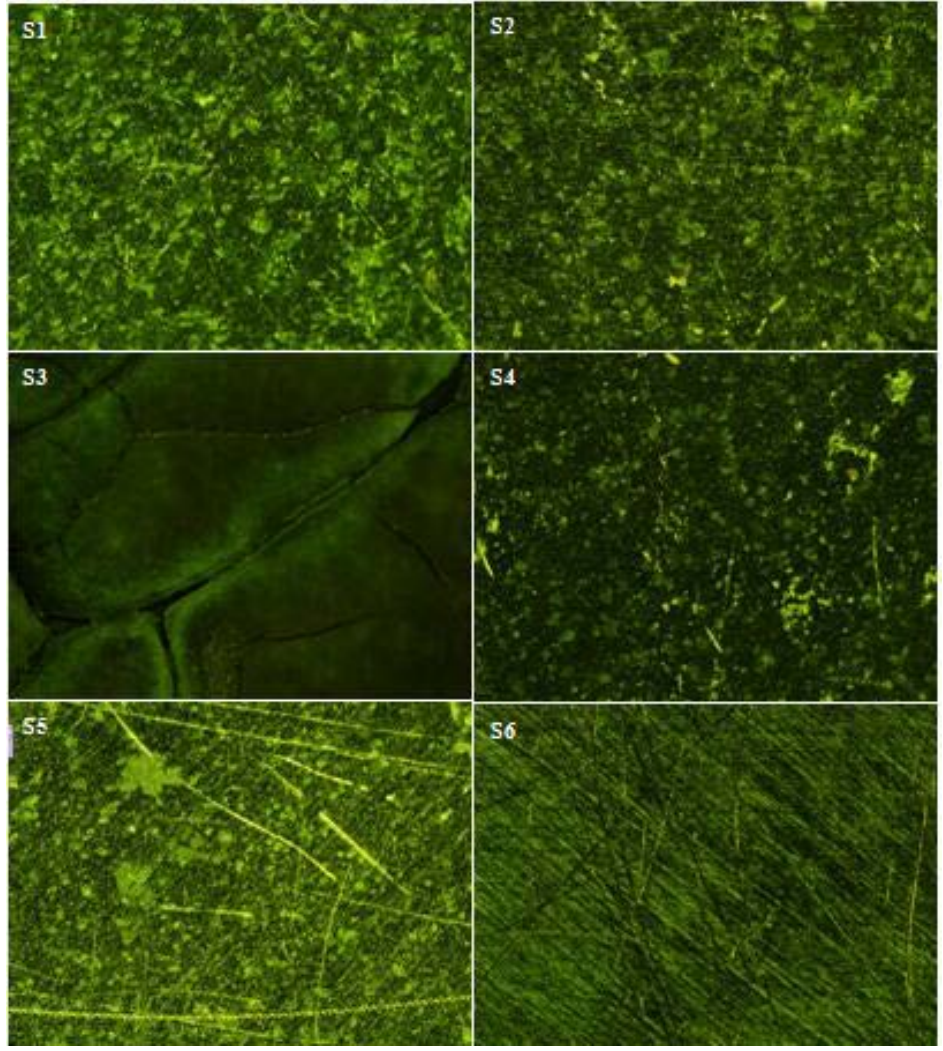

Fig 3 The cell state diagram of the surface of the metal material in the culture medium.

\subsubsection{Characteristic comparison of the metal materials on the culture medium}

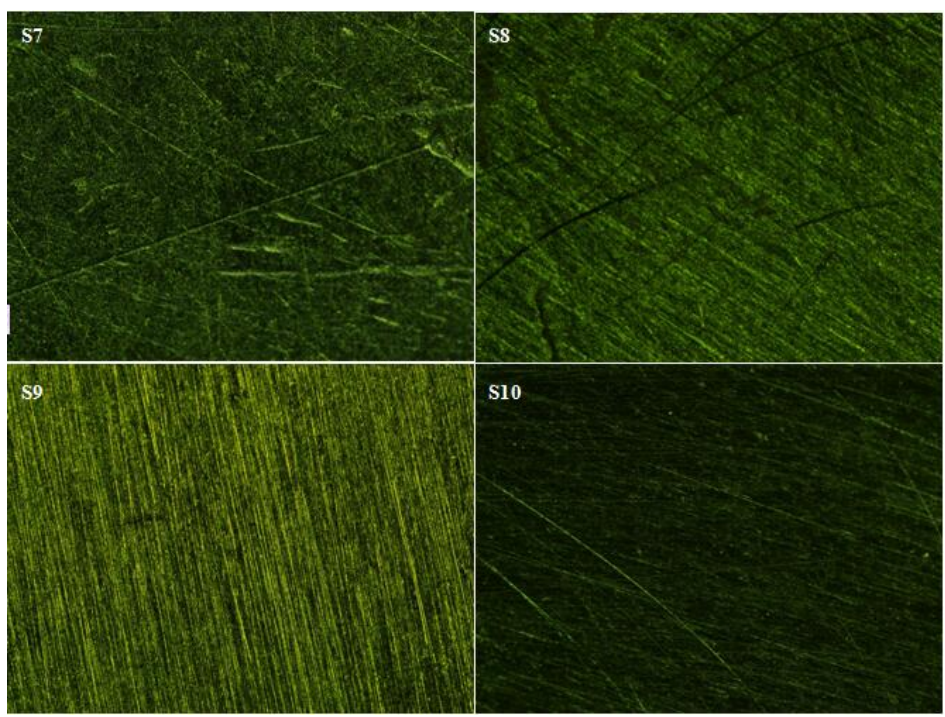

Fig 4 Comparison chart of the bottom of the metal material in the culture medium.

The four materials with good biocompatibility, including Specimen M, TNZT-1, TNZT-2 and TNZT-3, were selected for further analyses as shown in Fig 4. After the polishing and sterilizing process, the surface of Specimen $M$ became smooth, but some scratches were also generated. Additionally, the minor cracks appeared on the surface of TNZT-2 and TNZT-3. However, the surface of TNZT-1 became very glossy without obvious cracks. compared with Specimen M, 
TNZT-2 and TNZT-3, the TNZT-1 alloy material exhibited the best performance in the culture medium. Therefore, TNZT-1 was only selected for further research and analyses.

\subsection{Reduction process of TNZT alloy:}

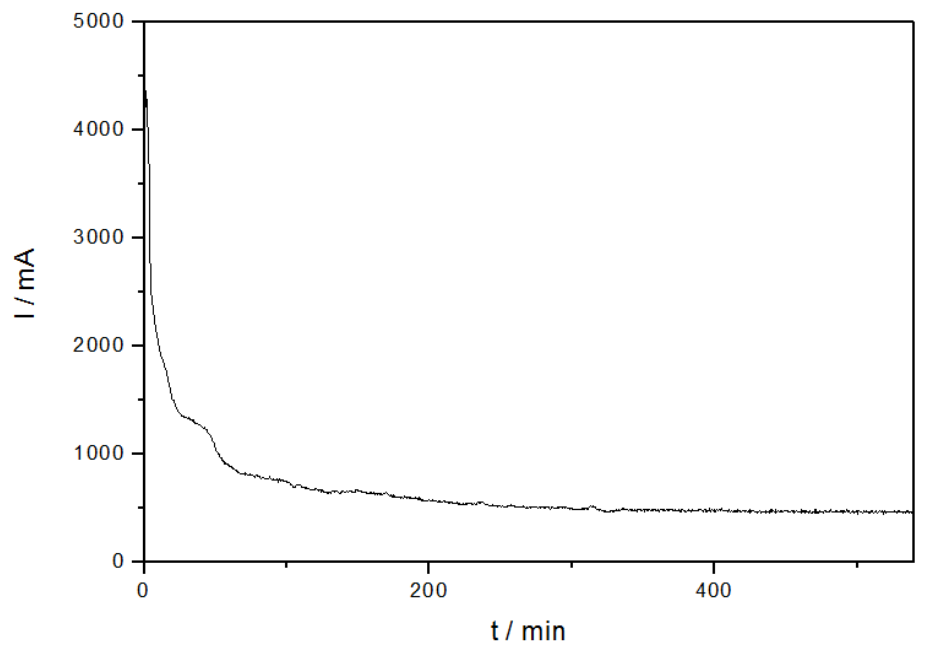

Fig 5 I-T curve in the process of TNZT-1 alloy prepared by electrolysis (electrolysis temperature: $900^{\circ} \mathrm{C}$, electrolysis time: $20 \mathrm{~h}$, cell voltage: $\left.3.0 \mathrm{~V}\right)$.

To further understand the electro-reduction process for the synthesis of TNZT-1, we measured the current-time curve during the electrolysis at the optimal experimental conditions (time: $20 \mathrm{~h}$, temperature: $900{ }^{\circ} \mathrm{C}$, voltage: $3.0 \mathrm{~V}$ ). The corresponding current-time curve was recorded in Fig 5. The current-time curve went though three stages, which including a fast initial improvement to a peak, then a following slower decrease, and finally a small but stable current. It is worth noting that the initial current improvement could be accounted for by the charge (electrons + ions) transfer reactions occurring at the three-phase lines, which was commonly observed in previous work [14].

\subsection{SEM and EDX analysis of the TNZT alloys :}

The reduction process of $\mathrm{TiO}_{2}, \mathrm{ZrO}_{2}, \mathrm{Ta}_{2} \mathrm{O}_{5}$ and $\mathrm{Nb}_{2} \mathrm{O}_{5}$ in constant depressed groove has been reported by the domestic and foreign counterparts $[15,16]$. The experimental results demonstrated that the calcium ions were embedded to form the perovskite structure in the reduction process. It also corresponds to the multiple stages of the current-time curve in Fig 5. The exact proportion of different elements of TNZT-1 was hardly obtained by EDX investigation, as shown in Table 2. This is because the peak of the $\mathrm{Nb}$ element coincided with the portion peak of the $\mathrm{Zr}$ element in the spectrums. Oxygen element on the outer surface and the part closing to the outer surface of the alloy was not detected. Taking into account the impact of the $\mathrm{Nb}$ element, the results of two tests indicated that the various contents of the alloy is similar (Table 2). However, the oxygen content in the central region of the alloy interface is about $6.10 \mathrm{wt} \%$. This is mainly results from the following two aspects: firstly, it is difficult that the elements $\mathrm{Nb}$ and $\mathrm{Zr}$ on the surface layer of the alloy spread to the inside layer due to high compaction and low porosity; secondly, the internal oxygen elements of the alloy are not completely deoxidized to be removed from the inside layer. Therefore, the oxygen elements of TNZT-1 was 3200 ppm, which mainly comes from its interior.

Table 2 EDX results for the TNZT-1

\begin{tabular}{|l|l|l|l|l|}
\hline & $\mathrm{Ti}(\mathrm{Wt} \%)$ & $\mathrm{Zr}(\mathrm{Wt} \%)$ & $\mathrm{Ta}(\mathrm{Wt} \%)$ & $\mathrm{O}(\mathrm{Wt} \%)$ \\
\hline 1 & 81.05 & 15.07 & 3.87 & Not Dectected \\
\hline 2 & 82.04 & 15.24 & 2.72 & Not Dectected \\
\hline
\end{tabular}

Based on the analysis of the above-mentioned experimental results, a reasonable process scheme of the molten salt electrolysis of solid oxides is provided. The metal layers of the product were formed on the surface in short time. The inner layer was mainly composed of perovskite and 
suboxides. Then, with the increasing of electrolysis time, the metal $\mid$ oxide $\mid$ molten salt threephase lines further expended to the depth of the inner layer, the inner layer of the products included variety phases (metals, oxides, perovskite, etc.). Finally, the mixture could be reduced to form the pure alloy.

\section{Conclusion}

In summary, Ti-12.5Zr-2.5Nb-2.5Ta (TNZT-1) with high biocompatibility and corrosion resistance was successfully prepared by solid oxide electrolysis in the molten salt. The mechanism of the reduction process was discussed by constant cell voltage electrolysis, indicating that the molten salt electrolysis route was of high current efficiency and low-energy consumption. The experimental results confirmed that TNZT-1 was the good biomedical implant materials, which represented a small scale functional material applied to the preparation of metal. Of course, we still need to study and solve many problems, such as further precise control of alloy elements in the products, reducing the oxygen content of the alloy, etc., which was aimed at improving the biocompatibility, biomechanical compatibility and biological binding of the alloy materials. We believe that the titanium alloy is a very promising candidate for artificial joints and open a new avenue to design and synthesize cost-affordable composite materials for medical implant application.

\section{Acknowledgement}

We acknowledge the financial support from the Natural Science Foundation of Hubei Province of China (Grant No.B2017210).

\section{References}

[1] Qian, J. J. Rare Earth 2001, 25. 303.

[2] Li, Z. H.; Wu, Y. S.; Liu, M. Journal of Chongqing Institute of Technology 2006, 5, 42.

[3] Yu, S. R. Mater. Sci. Eng. 2000, 18, 131.

[4] Qian, J. H. Rare Earth 2001, 24, 218.

[5] Long, M.; Rack, H. J. Biomaterials 1998, 19, 1621.

[6] Trill, E. A. J.Mater.Sci:Materials in medicine 2001, 12, 283.

[7] Glowacki, B. A.; Fray, D. J.; Yan, X. Y.; Chen, G. Z. Physica C 2003, 387, 242.

[8] Yan, X. Y.; Fray, D. J. Adv. Funct. Mater. 2005, 15, 1757.

[9] Muir Wood, A. J.; Copcutt, R. C.; Chen, G. Z.; Fray, D. J. Adv. Eng. Mater. 2003, 5, 650.

[10] Chen, G. Z.; Fray, D. J.; Farthing, T. W. Nature 2000, 407, 361.

[11] Wu, T.; Jin, X. B.; Xiao, W.; Liu, C.; Wang, D. H.; Chen, G. Z. Phys. Chem. Chem. Phys. 2008, 10, 1809.

[12] Prigent, H.; Pellen-Mussi, P.; Cathelineau, G.; Bonnaure-Mallet, M. Cell Proliferation on Titanium-tantalum 1998, 2, 200.

[13] Qiu, G. H.; Wang, D. H.; Jin, X. B.; Chen, G. Z. Electrochim. Acta 2006, 51, 5785.

[14] Wu, T.; Jin, X. B.; Xiao, W.; Hu, X. H.; Wang, D. H.; Chen, G. Z. Chem. Mater. 2007, 19, 153.

[15] Qiu, G. H.; Feng, X. H.; Liu, M. M.; Tan, W. F.; Liu, F. Electrochim. Acta 2008, 53, 4074.

[16] Yan, X. Y.; Fray, D. J. Metall. Mater. Trans. B 2002, B33, 685. 